\title{
Toma de Decisiones Motoras: una revisión sobre sus bases neurológicas
}

\section{Motor Decision-Making: a review of its neurological basis}

Pág. 9,11

Recibido: $11-08-2020$

Aceptado: 26-08-2020

Dra. Katherine Dawley Sandoval ${ }^{1}$

1.Médico General, Trabajador Independiente, San José Costa Rica

\section{RESUMEN:}

El proceso de toma de decisiones motoras se lleva a cabo diariamente, con el fin de seleccionar los pasos necesarios para realizar un movimiento determinado. Se ha identificado ciertas áreas cerebrales que trabajan en conjunto durante los diferentes puntos de selección del objetivo, del movimiento y del efector muscular.

El área interparietal, la corteza prefrontal, el área parietal de alcance y otros circuitos frontoparietales, son algunos de los responsables de la coordinación precisa que implica la complejidad del proceso; sin embargo, todavía faltan muchas estructuras contribuyentes por dilucidar.

Se presenta un recopilado sobre los diferentes procesos experimentales que se han llevado a cabo a lo largo de los últimos años y, a partir de los cuales se ha definido la participación de las diferentes estructuras encefálicas en el proceso de toma de decisiones motoras.

\section{PALABRAS CLAVE:}

toma de decisiones motoras, corteza prefrontal, corteza parietal, circuito frontoparietal.

\section{ABSTRACT:}

Motor decision-making is a process that takes place in everyday tasks to select the needed steps to make a specific movement. There are some brain areas that work together during the different stages of target selection, movement selection and muscular effector selection.

The intraparietal area, the prefrontal cortex, the reach parietal region and other frontoparietal networks, are some of the responsible structures that coordinate the complexity of the process; however there are still so many contributors that haven't been discovered yet. This is a summary about the different experimental processes in 
the last years, that have determined the brain structures that participate in the motor decisionmaking.

\section{KEY WORDS}

motor decision-making, prefrontal cortex, parietal cortex, frontoparietal network.

\section{INTRODUCCIÓN}

Las tareas motoras realizadas en la vida diaria involucran secuencias de acciones que se llevan a cabo para alcanzar un objetivo (1). El proceso de toma de decisión determina qué movimiento realizar, cuándo y cómo realizarlo; y está influenciado directamente por los cambios dinámicos que ocurren en el ambiente (1). Además, se debe coordinar el movimiento, su ejecución, el costo y la ganancia de realizarlo (1).

La escogencia o la toma de decisión se hace en concordancia con su probabilidad de llevar hacia el mejor resultado en términos de eficiencia biológica (2). Por lo tanto, la habilidad para predecir resultados futuros es crítica y determinante para todas las formas de toma de decisión: el cerebro debe estimar las consecuencias de cada opción de escogencia (2). Ya que muchos eventos son azarosos, la mayoría de las decisiones son realizadas con base en información muy limitada acerca de sus consecuencias; por lo tanto, se maneja un grado de incertidumbre (2).

Existe redes neuronales que incluyen estructuras corticales y subcorticales que minimizan esta incertidumbre, procesando la información que se encuentra disponible en el momento y la experiencia acumulada (2).
De acuerdo con la literatura, la experiencia consciente de la intención de la acción motora en humanos, se da de 200 a 500 ms después de que inició el planeamiento del movimiento a nivel cerebral (2). Por lo tanto, existe conexiones neuronales a nivel "inconsciente" que trabajan de manera autónoma y que son las protagonistas del proceso de toma de decisión. A continuación, se presenta un recopilado sobre los diversos experimentos que han contribuido a la identificación de las áreas cerebrales implicadas en el proceso de toma de decisiones motoras.

\section{SELECCIÓN DEL OBJETIVO}

La generación de un movimiento se ha conceptualizado en varias fases: una fase de planeamiento premotora, la fase motora en sí, y una fase de control del movimiento que se está realizando $(1,2)$.

Las acciones dirigidas a un objetivo no están restringidas a los comportamientos voluntarios conscientes exclusivamente, porque se basa más en las aferencias sensoriales que en las intenciones conscientes (2).

Las decisiones que se concentran en acciones motoras "goal-directed" o "dirigidas hacia un objetivo", difieren de otro tipo de acciones en el grado que involucran los comandos "topdown" o "de arriba hacia abajo" versus las señales sensoriales "bottom-up" o "de abajo hacia arriba" $(1,2)$. Las acciones dirigidas a un objetivo ya aprendidas, relacionan la información sensorial con las acciones motoras; y la parte que corresponde al proceso consciente de esto puede ser mínima (2). 
El primer paso para la selección del objetivo depende de la motivación: el cerebro evalúa si se tiene la necesidad de cambiar el estado actual para perseguir un objetivo o si no se necesita ningún cambio $(1,2)$. Esta evaluación se realiza constantemente para seleccionar solamente las acciones útiles, ya que existe una gama muy amplia de acciones disponibles (2).

Procesar las acciones accesibles se ha relacionado con el área motora suplementaria $y$, estas acciones se tienen que acoplar con estados internos corporales para que la acción sea ejecutable (2).

Si el proceso inicial termina en una acción que valelapenallevaracabo, se procedeala selección del objetivo per sé, lo cual tiene dos fases (2). En la primera fase, se asigna valor a las opciones disponibles (2). En la segunda fase, el contexto es considerado para ajustar estos valores (2). En este momento las representaciones neuronales de los posibles objetivos empiezan a competir a través de la inhibición mutua (2). El sistema de monitoreo determina quién gana la competencia $(1,2)$. Este sistema está formado por una red neuronal de áreas que selecciona la mejor estrategia motora, tomando en cuenta los datos sensoriales disponibles, los comportamientos aprendidos y los resultados de acciones previas $(1,2)$. La corteza orbitofrontal juega un papel central en asociar los estímulos a sus valores $(1,2)$. La corteza parietal posterior, juzga los recursos disponibles en el ambiente $(1,2)$. La corteza prefrontal lateral interactúa con la corteza cingular anterior, el sistema dopaminérgico, el área motora suplementaria y el polo frontal, y así, integra la información de las opciones disponibles, el contexto actual y los resultados de acciones previas $(1,2)$. El control de la atención se da por una red frontoparietal que media el procesamiento preferencial de la información relevante (2).

Los modelos más recientes de "retroalimentación óptima" u "optimal feedback control (OFC)" son enfáticosen laimportancia de la retroalimentación sensorial en el proceso de toma de decisiones motoras para llegar a un objetivo (1). La selección de una "política de control" que maneje la retroalimentación sensorial, se utiliza durante todo el proceso para generar los comandos motores y para el manejo de los errores durante la ejecución del movimiento; todo con la meta de alcanzar el objetivo seleccionado (1).

A nivel parietal, existe una zona conocida como el área lateral intraparietal, la cual responde a factores sensoriales, cognitivos y motores; y se relaciona directamente con el proceso de toma de decisiones motoras y ejecución de estas (3). En un estudio se registró la actividad a nivel del área intraparietal de monos, mientras éstos realizaban movimientos de alcance hacia un objetivo (4). Las neuronas del área intraparietal representaron la orientación del objeto y el tipo de agarre de este, indicando que el área intraparietal integra características tridimensionales (3D) de los objetos que se pueden alcanzar, con la información contextual del ambiente (4).

El área intraparietal recibe información de las áreas visuales, de corteza parietal y temporal; estas representan información especial y orientación de los objetos visibles (4). También recibe información de corteza prefrontal, la cual provee información del contexto (4). A su vez, el área intraparietal se conecta recíprocamente con la corteza premotora, de la cual sale información para el control de los movimientos de la mano por medio de proyecciones hacia la corteza motora primaria (4). Las neuronas del área intraparietal están continuamente activas: desde

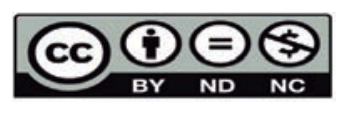


que se presenta el estímulo hasta la ejecución de la acción, o sea, representan no solamente el objeto, sino también, la información del contexto para seleccionar la acción apropiada (4).

Por su parte, los colículos superiores son necesarios para iniciar la orientación de los movimientos y para la toma de la decisión en el proceso motor hacia un objetivo específico (5). Existe evidencia que apoya la idea de que los colículos superiores participan en la selección de la respuesta motora, probablemente para llevar a una recompensa (5).

Además, los colículos superiores juegan un papel importante en la selección de targets u objetivos especiales para el movimiento, particularmente cuando el objetivo es indicado por estímulos sensoriales (6). Existen tres aferencias que influyen en el proceso: GABAérgica de la sustancia negra pars reticulata, colinérgica de la región parabraquial (núcleo pedúnculopontino tegmental y núcleo tegmental laterodorsal) y glutamatérgica de las regiones neocorticales (6). Estas señales convergen en la subdivisión más profunda de los colículos superiores (6). La combinación específica de referencias requeridas para alcanzar una meta específica de orientación hacia un objetivo, parece estar representada en la actividad del núcleo premotor del tallo cerebral, coordinado también por colículos superiores (6). Todas las aferencias que llegan a colículos superiores probablemente aportan información complementaria a la ya recibida por el input sensorial, lo cual se integra para seleccionar los targets u objetivos (6).

Pese a que el proceso de selección del objetivo se ha considerado tradicionalmente una parte fundamental para iniciar el movimiento, estudios recientes sugieren que, al existir varios targets u objetivos alcanzables, se desarrolla una competencia que evoca representaciones corticales sensoriales-motoras y especificación de movimientos, previo a la selección del objetivo $(1,6)$. Una de las posibles interpretaciones de estos hallazgos, es que el sistema motor prepara planes de movimiento para potenciales targets u objetivos, previo a la selección del objetivo en específico $(1,6)$.

En un estudio con monos, se ve que cuando se les presenta dos targets o potenciales objetivos para alcanzar, primero el sistema motor representa las opciones y posteriormente refleja la selección entre ellas (7). Cuando los dos targets potenciales se presentan, muchas células "relacionadas con la tarea" o "taskrelated" y sintonizadas con la dirección, en la corteza premotora dorsal, realizan descargas eléctricas si uno de los targets estaba cerca de la dirección preferida (7).

\section{SELECCIÓN DEL EFECTOR}

Existe una interacción entre las propiedades intrínsecas de una extremidad y el grado de control que demanda el ambiente que la rodea (8). Esta interacción puede influenciar la selección de las acciones motoras (8). El proceso de decisión entre las acciones motoras es muy dependiente del contexto, es gradual e influenciado por las propiedades dinámicas ambientales, en relación con las propiedades intrínsecas del aparato motor (8).

Constantemente se toman decisiones con respecto a utilizar alguno de los dos brazos (9). Generalmente se utiliza más la mano dominante para alcanzar objetos y realizar tareas complejas, y la escogencia se ha explicado en términos de recompensas recibidas (9). Por 
ejemplo, la diferencia de selección entre los dos brazos se puede dar por la recompensa: éxito del movimiento (9). En un experimento con 26 personas diestras, se determinó que la mano derecha se escogió en mayor porcentaje, tanto para objetos pequeños como grandes (9). Se vio que el esfuerzo juega un papel importante en la elección del brazo (9). La probabilidad de la escogencia del brazo derecho aumentó con objetos pequeños y demostró un menor esfuerzo motor esperado y una mayor tasa de éxito (9). Se demostró que la elección del brazo depende de la diferencia en el esfuerzo esperado entre el lado derecho y el izquierdo (9). Esto concuerda con otros estudios que han determinado que el Sistema Nervioso Central toma en cuenta las propiedades biomecánicas de cada brazo para realizar la escogencia de este (9).

Se ha propuesto que el hemisferio izquierdo juega un papel especial en el proceso motor $y$ que existen diferencias cognitivas entre los dos hemisferios (10). En un estudio de Coelho y colegas, se propuso que existen dos sistemas basados en la dominancia de los hemisferios cerebrales: un sistema dominante para la coordinación de las extremidades y otro sistema no dominante, que se encarga de estabilizar la posición de estas (10). Coelho estudió este fenómeno, colocándole a personas diestras, diversos objetos al lado derecho, al lado izquierdo y en línea media, para que realizaran movimientos de alcance (10). Se comprobó que los objetos que se encontraban en línea media tuvieron más acercamientos e intentos de alcance, por medio de la mano dominante (10). Además, las trayectorias del brazo dominante fueron más rectas y se demostró un mayor torque comparado con el brazo no dominante (10). Este estudio demostró que existe una relación entre las asimetrías sensoriales-motoras en la dinámica de la coordinación y la decisión de las personas de cómo realizar un movimiento (10). En otro estudio, se tomó a 12 participantes, a los cuales se les sentó en una silla en un cuarto oscuro, con los brazos apoyados a los lados, las manos en posición neutra y con los pulgares encima de cilindros que tenían sensores de fuerza (11). Los participantes se encontraban a $80 \mathrm{~cm}$ de una pantalla negra a nivel de los ojos y en la pantalla se mostraban 3 LEDs (verde central y amarillos laterales, los cuales correspondían a las señales de respuesta) (11).

El estudio consistió en que de acuerdo con las señales visuales que recibían los participantes, debían presionar al lado derecho o al izquierdo, según correspondiera (11). Los participantes realizaron una sesión de entrenamiento, y posteriormente, se realizó estimulación magnética transcraneal hacia la corteza motora izquierda, como sesión experimental (11). El realizar la estimulación en hemisferio izquierdo, creó un potencial motor evocado a nivel de la mano derecha, como respuesta temprana (11). Esto refleja una modulación en la excitabilidad de la vía motora (11). Esta activación motora temprana sugiere que el potencial de acción puede inicialmente ser implementado previo a la acción motora en sí, que se seleccionó (11).

La activación refleja un mecanismo anticipatorio relacionado con la preparación de potenciales de acción para posteriormente efectuar un movimiento de respuesta a una tarea (11). Previo a la implementación de la acción seleccionada, la amplitud del potencial motor evocado primero incrementó con el estímulo visual, pero solamente para la mano que estaba potencialmente envuelta en la acción que se iba a producir (11). 


\section{SELECCIÓN DE LAACCIÓN}

Las neuronas de la corteza premotora dorsal representan múltiples acciones que compiten hasta que se determina una ganadora (2). Dentro de todo el proceso, existe una red neuronal que puede inhibir la acción (2). El giro frontal inferior (sobre todo el derecho), el área motora suplementaria, el núcleo subtalámico y el estriado, forman parte de esta red (2).

Tanto el nivel como la fuente de la incertidumbre en un movimiento tienen un efecto significativo en el desarrollo de la planificación de la acción durante la toma de decisiones motoras (12). Las personas efectúan más rápidamente respuestas esperadas, que respuestas inesperadas, inclusive cuando la tarea a realizar sea sencilla (13). Esto sugiere que los tiempos de ejecución motora son más rápidos cuando la respuesta requerida puede ser anticipada (13). Se espera que la estrategia motora escogida y posteriormente aplicada, sea la que maximice la ganancia esperada (14).

Los circuitos frontoparietales, parecen estar involucrados en decidir y planear las acciones (15). Las neuronas en ambas cortezas muestran actividad relacionada con movimientos intencionados (15). La atención puede influenciar las aferencias para los procesos de decisión (15). Algunos estudios proponen que el área intraparietal lateral contribuye al control de la atención, pero, además, está involucrada en procesos de representación de valor, planeamiento de movimientos oculares, predicción, categorización, funciones cognitivas, reconocimiento de formas y reconocimiento temporal (15).

Las propiedades biomecánicas de las diferentes acciones también pueden ejercer una fuerte influencia en el proceso de toma de decisión de un movimiento (16). En un estudio se examina los factores que llevan a tomar las decisiones motoras que se efectúa durante un movimiento en curso (17). En esta investigación, no se encontró consistencia en la influencia del costo biomecánico del movimiento y la decisión de cambiar de objetivo de alcance (17). Los sujetos no tomaron decisiones motoras que minimizaran el costo biomecánico, sino, tomaron las decisiones que se acoplaran con los objetivos propuestos, por ejemplo, mantener la posición y la velocidad de su mano igual a la de un objeto en movimiento (17). Además, el tiempo de reacción para cambiar la decisión, mientras ya ejecutaba un movimiento, se encontró dentro del rango normal de tiempos de reacción para la toma de decisiones motoras simples (como el ejercicio de iniciar un movimiento con una señal de "go") (17). Probablemente, en este estudio, la meta u objetivo consistía en cumplir con el movimiento a cabalidad; lo cual coloca al costo biomecánico en un lugar secundario (17).

En contraposición a lo anterior, en otro estudio se les pidió a los sujetos que escogieran entre dos movimientos de brazo de diferente amplitud o duración, que eran realizados contra diferentes niveles de resistencia (18). Se registró que la amplitud del movimiento no influyó en el esfuerzo, pero la fuerza y la duración, sí; ya que los movimientos con una duración mayor se perciben como movimientos de mayor esfuerzo que los movimientos de duración más corta, todo esto contra la misma resistencia (18). Además, se vio que, los sujetos prefieren movimientos hacia la línea media corporal, que los movimientos que se alejan de ella (18).

De acuerdo con estos hallazgos, se infiere que los sujetos toman en cuenta las propiedades biomecánicas de los efectores motores en su 
decisión, de hecho, las toman más en cuenta que otro tipo de costo, por ejemplo, el metabólico, porque influyen directamente en el control motor (18).

Cuando los humanos toman una decisión motora de alcance, entre dos objetos, se tiende a seleccionar el movimiento que tenga menor costo bioenergético y mecánico (1). La predicción del esfuerzo asociado con los movimientos posibles, se integra muy rápidamente e influencia las decisiones motoras en corteza frontal en aproximadamente $200 \mathrm{~ms}$ (1). Representaciones del valor de la acción se han reportado a nivel de giro cingular anterior, estriado y corteza parietal posterior, en estudios electrofisiológicos en monos; $y$, además, en la corteza prefrontal ventromedial en humanos (19).

\section{PROCESO DE DECISIÓN Y ÁREAS CORTICALES INVOLUCRADAS}

Cuando el cerebro está definiendo las secuencias de acciones motoras para completar una tarea, tiene que monitorizar e integrar la información acerca de acciones recientes y sus resultados (20). Existe evidencia que indica que múltiples regiones en la corteza frontal, contribuyen a la coordinación adecuada de una secuencia de acciones (20). Se estudió en monos la aplicación de secuencias de movimiento, haciendo que los primates fueran tomando decisiones al imitar una secuencia dada; sin embargo, se trataba de una secuencia previamente aprendida y el patrón que debían imitar, era incorrecto (20). Se toma mediciones de 485 neuronas de la corteza prefrontal del macaco, las cuales reflejaron la cinemática de los movimientos y sus aspectos secuenciales (20). Cuando los monos descubrían la secuencia correcta de movimientos, la actividad neuronal gradualmente se hacía más similar a los patrones observados en los monos que realizaban la secuencia correctamente (20). La teoría de la "decisión óptima de movimiento" requiere el valor de un resultado y la seguridad subjetiva de que ese resultado se va a obtener (20). Trabajos previos muestran que la actividad neuronal que existe en la corteza parietal puede reflejar la confianza del mono en su decisión (20).

También, se examinó el área dorsolateral de la corteza prefrontal de monos, haciéndolos seguir con un cursor, un camino para alcanzar un objeto o una meta (21). Se vio que la actividad neuronal de esta zona refleja las acciones planeadas que se debían realizar con el cursor, en vez de los movimientos del brazo que se requieren para realizar estas acciones; por su parte, la actividad neuronal en la corteza motora primaria refleja los movimientos del brazo per sé (21). Se encontró que las neuronas en el área lateral de la corteza prefrontal sufrían cambios en su actividad, durante el período preparatorio en el que los monos planeaban los múltiples pasos que llevaban a realizar un comportamiento motor (21). Esa actividad neuronal refleja los movimientos futuros que se van a realizar para alcanzar un objeto, en este caso, los movimientos del cursor (21). Sólo el 9\% de las neuronas prefrontales reflejó los movimientos del brazo durante el período preparatorio (21).

A nivel de la corteza parietal posterior, existen dos subpoblaciones neuronales: una que se proyecta hacia el estriado y otra que se proyecta hacia la corteza motora secundaria en su zona posterior (22). En un estudio realizado en ratas, se encontró que el inactivar las neuronas que se proyectan hacia el estriado, debilita el sesgo historia-dependiente o la tendencia de la historia de movimientos ya conocidos, de influenciar a la nueva decisión (22). En contraste, al inactivar las neuronas que se proyectan hacia

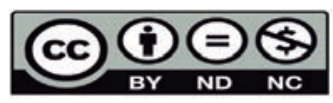


la corteza motora secundaria, se altera la cinemática de los movimientos (22). Estas dos zonas en la corteza parietal posterior reciben información de diferentes áreas corticales, pero en proporciones distintas (22). Esto sugiere que estas subpoblaciones forman vías paralelas distintas (22). La vía hacia la corteza motora secundaria puede ser crítica para la preparación adecuada de un movimiento, tomando en cuenta su dirección, amplitud y velocidad (22).

Las neuronas que se encuentran a nivel de la corteza parietal posterior no solo están envueltas en formar planes de movimiento, sino en monitorizar la meta del movimiento y el estado dinámico de la trayectoria durante su ejecución. (23). Este monitoreo es importante para controlar y corregir el error (23).

La "región parietal de alcance" o "parietal reach region" codifica señales cognitivas relacionadas con el movimiento de alcance del brazo, la dirección del movimiento y el valor asociado con la ejecución de esas acciones (24). La corteza parietal posterior está involucrada en la transformación de señales sensoriales en acciones motoras concretas (24). Células de la corteza parietal posterior, también muestran especificidad dependiendo del efector del movimiento, por ejemplo: el ojo o la mano (25). Células en el área intraparietal lateral son selectivas para movimientos sacádicos oculares y células en la "región parietal de alcance" o "parietal reach region", selectivas para movimientos de alcance específicamente; esto bajo condiciones de estímulos idénticos (25). Todavía existe mucha controversia en poder determinar si la corteza parietal posterior refleja el resultado de una decisión que se tomó en áreas corticales superiores o si juega un papel causal en la deliberación del proceso para la toma de decisiones primarias (25).
Existe un estudio donde se tomó registro del área premotora dorsal en la corteza frontal y de la región parietal que se encarga del "alcance o agarre" conocida como "parietal reach region" en la corteza parietal (26). Estas dos áreas se estudiann para identificar un circuito neuronal que explicara la decisión de "hacia dónde" realizar el movimiento de alcance (26). Se entrenó a dos monos para que realizaran una tarea con libre escogencia y otra tarea siguiendo instrucciones (en ambas, el mono realiza una secuencia de movimientos de alcance hacia targets visuales y como premio recibe jugo) (26). En la tarea de libre escogencia, los tres targets eran círculos visuales idénticos y el mono podía alcanzar el objeto en cualquier secuencia de movimiento (26). En la tarea que tenía instrucciones, se encontraba: un círculo, un cuadrado y un triángulo, y el mono tenía que seguir una secuencia fija (26). Se analizó el "local field potential (LFP)" o potencial local de campo; su actividad mide los potenciales sinápticos en una población de neuronas cerca de un electrodo (26). También se analizó la "coherencia de espiga-campo", que mide qué tan bien la actividad del LFP se predice por medio de los potenciales de acción (26). La coherencia de "spike-field" entre la corteza premotora y la parietal, fue más prevalente durante la libre escogencia, lo que sugiere que esta correlación está asociada con tomar una decisión motora (26).

La coherencia es débil durante las tareas instruidas (26). La corteza prefrontal, la frontal medial y la corteza cingular, pueden modular la coherencia frontoparietal, durante la toma de decisiones (26). La información podría ir de frontal hacia parietal y luego devolverse, como una especie de circuito sináptico de retroalimentación y de aumento de la comunicación que refleja la toma de la decisión (26). 
En un estudio donde se realizó estimulación eléctrica en una neurocirugía con paciente despierto, se reportó que, al estimular la corteza parietal posterior, el efecto consiste en que los pacientes intenten moverse y que reporten haberse movido, inclusive en la ausencia de repuestas motoras (27). Mientras que, la estimulación de la corteza premotora inicia movimiento de boca y extremidades que no fueron detectados conscientemente por los pacientes (27). El estudio sugiere que la intención motora y la consciencia, son consecuencias de un incremento en la actividad parietal, previo a que se ejecute el movimiento (27).

Se ha visto que neuronas en la corteza prefrontal del macaco, sirven como "marcadores" de inicio y de finalización de algunas secuencias motoras (28). En diversas subpoblaciones neuronales prefrontales, se han registrado espigas de actividad que ocurrían al inicio y al final de una secuencia de un movimiento oculomotor sacádico (28). La corteza parietal posterior también podría estar implicada en participar en el ajuste de los comandos motores necesarios para la adaptación a un ambiente mecánico nuevo (29).

Asimismo, el papel de contexto también influencia la toma de decisiones motoras (30). En un estudio se juzgó el efecto de la interacción con oponentes en la toma de decisiones motoras, con la predicción de que las intenciones 0 acciones de otras personas, pueden influenciar el sistema motor de los sujetos (30). Las decisiones motoras óptimas se promovieron con la competencia contra un oponente (30). Las decisiones motoras de los sujetos se vieron influenciadas de manera no lineal por las decisiones de los oponentes, esto quiere decir que, si los oponentes realizan una mejor tarea motora que los sujetos, éstos últimos, incrementan la calidad de su tarea (30).
Sin embargo, si los oponentes realizan una peor tarea motora, los sujetos no cambian su objetivo ni la calidad de su tarea (30). O sea, adaptativamente alteran sus decisiones de acuerdo con las decisiones de sus oponentes, pero no los imitan (30). También se ha sugerido un papel importante para la corteza prefrontal dorsolateral en modular la selección de la acción, en decisiones motoras que son tomadas en contextos de riesgo (31). En un estudio se encontró que el comportamiento de riesgo en decisiones motoras disminuye con la activación de esta área a nivel derecho y con la desactivación a nivel izquierdo (31). Los participantes de este experimento demostraron estrategias más conservadoras, precisamente para evitar los riesgos (31).

\section{CONCLUSIÓN}

Existe múltiples interacciones sensoriales y motoras que se dan durante el proceso de toma de decisiones, las cuales ocurren en múltiples niveles de planeamiento motor y de acción.

Para controlar estas acciones, el Sistema Nervioso Central utiliza transformaciones sensoriales-motoras que permiten convertir información sensorial/aferente, en información motora/eferente. Estas transformaciones se dan a nivel de múltiples áreas corticales y subcorticales que se integran a nivel funcional, con el objetivo de tomar la decisión más correcta para llevar a cabo el proceso de secuencias de movimientos con el que se alcance la meta deseada.

Pese a las conexiones que se han descubierto y a las hipótesis que se han planteado en diversos estudios experimentales, todavía falta describir muchos mecanismos específicos que involucren todo el proceso de toma de decisiones motoras. 


\section{REFERENCIAS}

1. Gallivan JP, Chapman CS, Wolpert DM, Flanagan JR. Decision-making in sensorimotor control. Nat Rev Neurosci [Internet]. 2018;19(September):519-34. Disponible en: http://dx.doi. org/10.1038/s41583-018-0045-9.

2. Mirabella G, Lebedev MA. Decision Making: Neural Mechanisms Interfacing to the brain's motor decisions. J Neurophysiol. 2017; 117:1305-19.

3. Huk AC, Katz LN, Yates JL. The Role of the Lateral Intraparietal Area in (the Study of) Decision Making. Annu Rev Neurosci. 2017; 40:349-72.

4. Baumann MA, Fluet M, Scherberger H. Context-Specific Grasp Movement Representation in the Macaque Anterior Intraparietal Area. J Neurosci. 2009;29(20):6436-48.

5. Felsen G, Mainen ZF. Midbrain contributions to sensorimotor decision making. J Neurophysiol. 2012; 108:135-47.

6. Wolf AB, Lintz MJ, Costabile JD, Thompson JA, Stubblefield EA, Felsen G. An integrative role for the superior colliculus in selecting targets for movements. J Neurophysiol. 2015; 114:2118-31.

7. Cisek P, Kalaska JF. Neural Correlates of Reaching Decisions in Dorsal Premotor Cortex: Specification of Multiple Direction Choices and Final Selection of Action. Neuron. 2005; 45:801-14.

8. Cos I, Medleg F, Cisek P. The modulatory influence of end-point controllability on decisions between actions. J Neurophysiol. 2012; 108:1764-80.

9. Schweighofer N, Xiao Y, Kim S, Yoshioka T, Gordon J, Osu R. Effort, success, and nonuse determine arm choice. J Neurophysiol. 2015; 114:551-9.

10. Coelho CJ, Przybyla A, Yadav V, Sainburg RL. Hemispheric differences in the control of limb dynamics: a link between arm performance asymmetries and arm selection patterns. J Neurophysiol. 2013; 109:825-38.

11. Tandonnet C, Garry MI, Summers JJ. Decision making and action implementation: Evidence for an early visually triggered motor activation specific to potential actions. Psychophysiology. 2013;50(7):701-10.

12. Krüger M, Hermsdörfer J. Target Uncertainty During Motor Decision-Making: The Time Course of Movement Variability Reveals the Effect of Different Sources of Uncertainty on the Control of Reaching Movements. Front Psychol. 2019;10(January):1-13.

13. Starns JJ, Ma Q. Response biases in simple decision making: Faster decision making, faster response execution, or both? Psychon Bull Rev. 2017; 25:1535-41.

Trommersha J, Maloney LT, Landy MS. Decision making, movement planning and statistical decision theory. Trends Cogn Sci. 2008;12(July):291-7.

14. Andersen RA, Cui H. Intention, Action Planning, and Decision Making in Parietal-Frontal Circuits. Neuron [Internet]. 2009;63(5):568-83. Disponible en: http://dx.doi.org/10.1016/j.neuron.2009.08.028.

15. Cos I, Khamassi M, Girard B. Modelling the learning of biomechanics and visual planning for decision-making of motor actions. J Physiol. 2013; 107:399-408. 
16. Michalski J, Green AM, Cisek P. Reaching decisions during ongoing movements. J Physiol. 2020.

17. Morel P, Ulbrich P, Gail A. What makes a reach movement effortful? Physical effort discounting supports common minimization principles in decision making and motor control. Plos Biol. $2017 ; 15: 1-23$.

18. Wu S, MR D, Maloney LT. Motor Decision-Making. Brain Mapp An Encycl Ref. 2015; 3:417-27.

19. Averbeck BB, Sohn J, Lee D. Activity in prefrontal cortex during dynamic selection of action sequences. Nat Neurosci. 2006;9(2):276-82.

20. Mushiake H, Saito N, Sakamoto K, Itoyama Y, Tanji J. Activity in the Lateral Prefrontal Cortex Reflects Multiple Steps of Future Events in Action Plans. Neuron. 2006; 50:631-41.

21. Hwang EJ, Link TD, Hu YY, Neil KO, Hwang EJ, Link TD, et al. Corticostriatal Flow of Action Selection Bias. Neuron [Internet]. 2019; 104:1-15. Disponible en: https://doi.org/10.1016/j.neuron.2019.09.028.

22. Mulliken GH, Musallam S, Andersen RA. Forward estimation of movement state in posterior parietal cortex. Proc Natl Acad Sci U S A. 2008;105(24):8170-7.

23. Scherberger H, Andersen RA. Target Selection Signals for Arm Reaching in the Posterior Parietal Cortex. J Neurosci. 2007;27(8):2001-12.

24. Cui H, Andersen RA. Posterior Parietal Cortex Encodes Autonomously Selected Motor Plans. Neuron. 2007; 56:552-9.

25. Pesaran B, Nelson MJ, Andersen RA. Free choice activates a decision circuit between frontal and parietal cortex. Nature. 2008;453(May):406-10.

26. Desmurget M, Mottolese C, Sirigu A. Movement Intention After Parietal Cortex Stimulation in Humans. Science (80- ). 2009; 324:811-3.

27. Fujii N, Graybiel AM. Representation of Action Sequence Boundaries by Macaque Prefrontal Cortical Neurons. Science (80- ). 2003;301(August):1246-50.

28. Della-maggiore V, Malfait N, Ostry DJ. Stimulation of the Posterior Parietal Cortex Interferes with Arm Trajectory Adjustments during the Learning of New Dynamics. J Neurosci. 2004;24(44):9971-6.

29. Ota K, Tanae M, Ishii K, Takiyama K. Optimizing motor decision- making through competition with opponents. Sci Rep [Internet]. 2020;1-14. Available from: http://dx.doi.org/10.1038/ s41598-019-56659-6.

30. Ota K, Shinya M, Kudo K. Transcranial Direct Current Stimulation Over Dorsolateral Prefrontal Cortex Modulates Risk-Attitude in Motor. Front Hum Neurosci. 2019;13(September):1-11.

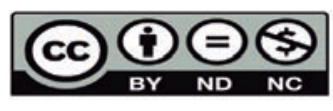

